Article

\title{
Fungal Diversity Analysis of Grape Musts from Central Valley-Chile and Characterization of Potential New Starter Cultures
}

\author{
Dinka Mandakovic ${ }^{1,2,+}$, Rodrigo Pulgar ${ }^{1,3,+}$, Jonathan Maldonado ${ }^{1,+}+\mathcal{D}$, Wladimir Mardones ${ }^{4,5}$, \\ Mauricio González 1,2,3, Francisco A. Cubillos $4,5, *$ (i) and Verónica Cambiazo ${ }^{1,2,3, *}$ \\ 1 Laboratorio de Bioinformática y Expresión Génica, Instituto de Nutrición y Tecnología de los \\ Alimentos (INTA), Universidad de Chile, Santiago 7810000, Chile; dinka.mandakovic@inta.uchile.cl (D.M.); \\ rpulgar@inta.uchile.cl (R.P.); jomaldon@gmail.com (J.M.); mgonzale@inta.uchile.cl (M.G.) \\ 2 FONDAP Center for Genome Regulation, Santiago 7810000, Chile \\ 3 Diagnofast SPA, Santiago 7810000, Chile \\ 4 Millennium Institute for Integrative Biology (iBio), Santiago 7500565, Chile; wladimirmardones2@gmail.com \\ 5 Departamento de Biología, Facultad de Química y Biología, Universidad de Santiago de Chile, \\ Santiago 9160000, Chile \\ * Correspondence: francisco.cubillos.r@usach.cl (F.A.C.); vcambiaz@inta.uchile.cl (V.C.) \\ † Current Address: GEMA Center for Genomics, Ecology and Environment, Universidad Mayor (D.M.); \\ Laboratorio de Genética y Genómica de Interacciones Biológicas, INTA, Universidad de Chile (R.P.); \\ Laboratorio de Biología de Sistemas de Plantas, Pontificia Universidad Católica de Chile (J.M.).
}

Received: 5 June 2020; Accepted: 22 June 2020; Published: 24 June 2020

\begin{abstract}
Autochthonous microorganisms are an important source of the distinctive metabolites that influence the chemical profile of wine. However, little is known about the diversity of fungal communities associated with grape musts, even though they are the source of local yeast strains with potential capacities to become starters during fermentation. By using internal transcribed spacer (ITS) amplicon sequencing, we identified the taxonomic structure of the yeast community in unfermented and fermented musts of a typical Vitis vinifera L. var. Sauvignon blanc from the Central Valley of Chile throughout two consecutive seasons of production. Unsurprisingly, Saccharomyces represented the most abundant fungal genus in unfermented and fermented musts, mainly due to the contribution of S. uvarum $(42.7 \%)$ and S. cerevisiae $(80 \%)$. Unfermented musts were highly variable between seasons and showed higher values of fungal diversity than fermented musts. Since microbial physiological characterization is primarily achieved in culture, we isolated nine species belonging to six genera of fungi from the unfermented must samples. All isolates were characterized for their potential capacities to be used as new starters in wine. Remarkably, only Metschnikowia pulcherrima could co-exist with a commercial Saccharomyces cerevisiae strain under fermentative conditions, representing a feasible candidate strain for wine production.
\end{abstract}

Keywords: grape must; high-throughput sequencing; non-conventional yeasts; Sauvignon blanc; Metschnikowia pulcherrima; starter culture fermentation; culture-dependent method

\section{Introduction}

During alcoholic fermentation of grape musts, sugars are transformed into ethanol and carbon dioxide by the actions of fermentative yeasts, mainly Saccharomyces cerevisiae. Wine fermentation is typically carried out by selected S. cerevisiae strains as microbial starters. Nevertheless, wine fermentation can be a spontaneous process, when it is carried out by the sequential action of different indigenous yeasts (including Saccharomyces or non-conventional yeasts), which are present in the must, and often 
in greater numbers than S. cerevisiae. In fact, non-conventional yeasts contribute to the first stages of fermentation and to the organoleptic characteristics of final wine [1]; however, the high efficiency of S. cerevisiae to convert sugars into ethanol and its ability to withstand the adverse stress conditions of fermentation results in the progressive death of other yeast species [2-4]. Although S. cerevisiae is the primary microorganism responsible to complete wine fermentation, at present, a re-evaluation of the role of non-conventional yeasts in winemaking and their use as selected starters in mixed fermentations with S. cerevisiae is being carried out [5]. In this context, non-conventional yeasts have the potential to positively contribute to the wine sensory profile through their distinctive production of secondary metabolites and fine-tune the ethanol and glycerol concentrations in wine [5,6].

In recent years, the development of high-throughput sequencing (HTS) provided a useful tool to describe bacterial and fungal communities present in berries, musts and in different stages of fermentation [7-10]. In particular, non-conventional yeasts have been characterized as a highly diverse population of microorganisms that can be influenced by numerous factors like grape variety, geographical area, climatic factors, sanitary status of grape berries and agronomic practices, among others [8,11-13]. Interestingly, the different yeast communities seem to be stably maintained at distinct viticulture regions; hence, the resulting biodiversity of grapevine-associated microbiota could potentially identify a vineyard, linking the wine characteristics to a terroir [14,15]. Therefore, a detailed taxonomic and functional analysis on the microbiota of grape musts could allow the identification of indigenous yeast strains with the potential to contribute to the regional profile of wines [16-19].

Even though Chile is currently the seventh largest wine-producing and fifth largest wine-exporting country of mostly medium-quality wines, studies of native microbial ecology in grape musts [20] or during the fermentation process [21] are scarce. To the best of our knowledge, no HTS studies on yeast diversity in combination with a culture-based approach have been conducted in unfermented and fermented musts from Chilean vineyards. In this study, the utilization of cultivation techniques coupled with high throughput internal transcribed spacer (ITS) sequencing allowed us to describe the fungal landscape of the indigenous wine yeasts from the variety of Vitis vinifera L var. Sauvignon blanc, the white variety with the largest Chilean wine growing area, with more than 15,000 hectares and $10.7 \%$ of the national surface in 2018 [22]. In addition, isolates were characterized in depth to describe their biotechnological potential as new starters in wine.

\section{Materials and Methods}

\subsection{Sample Collection, Processing, and Physicochemical Analyses}

The research site is located on the Central Valley of Chile (Curicó) and was surveyed throughout two consecutive seasons of production (years 2016 and 2017), in dependencies of Viña San Pedro $\left(35^{\circ} 05^{\prime} \mathrm{S}\right.$ and $\left.71^{\circ} 19^{\prime} \mathrm{W}\right)$. Samples were collected from unfermented (M) must of a typical Vitis vinifera L. var. Sauvignon blanc and from the fermented wine must at the end of the fermentation using a commercial Saccharomyces cerevisiae strain (EF; 30 days post M). Samples were obtained in triplicate from each fermentation step and were used separately as biological replicates. Sub-samples were used for: (1) community DNA extraction, which were placed into sterile plastic bags and immediately stored in dry ice for transport to the laboratory where they were frozen at $-80^{\circ} \mathrm{C}$ until DNA extraction, and (2) for fungal growth, which were placed into sterile plastic bags and immediately stored at $4{ }^{\circ} \mathrm{C}$ for transport to the laboratory where they were plated a few days after sampling.

The physicochemical data was obtained from Viña San Pedro domestic analyses that incorporated the Fourier transformed Infrared technology (FTIR) using a wine scan FT 120 Foss. All measurements were validated in agreement to the analyses proposed by the International Wine Organization.

\subsection{Microbiome DNA Extraction, PCR Amplification, and High-Throughput Sequencing}

Microbiome DNA was extracted using DNeasy Blood and Tissue Kit (Qiagen, Hilden, Germany) following manufacturer's instructions and including mechanic lysis of the samples using disruption 
spheres (FastPrep-24 MP). Extracted DNA was visualized in Tape Station 2200 (Agilent Technologies, Santa Clara, CA, USA) using Genomic DNA Screen Tape, according to the manufacturer's indications and quantified by fluorescent probes (Qubit Thermo Fisher Scientific, Waltham, MA, USA).

Internal transcribed spacer (ITS) was amplified using the primer set ITS1 (5'-TCCGTAGGTGAACCTGCGG-3') and ITS2 (5'-GCTGCGTTCTTCATCGATGC-3'), with a barcode in the forward primer. For the amplification, the kit HotStarTaq Plus Master Mix (Qiagen, Hilden, Germany) was used with the following conditions: $94{ }^{\circ} \mathrm{C} 3 \mathrm{~min}, 28$ cycles of $94{ }^{\circ} \mathrm{C} 3 \mathrm{~s}, 53{ }^{\circ} \mathrm{C} 4 \mathrm{~s}$, and $72{ }^{\circ} \mathrm{C} 1 \mathrm{~min}$, followed by an elongation phase of $72{ }^{\circ} \mathrm{C} 5 \mathrm{~min}$. PCR products were examined in agarose gels ( $2 \%)$. Samples were purified using Agencourt AMPure XP (Beckman Coulter, Brea, CA, USA). DNA libraries were constructed following the protocol TruSeq DNA sample preparation (Illumina, San Diego, CA, USA). Sequencing was performed by MrDNA Next Generation Sequencing Service Provider (Shallowater, TX, USA) on Illumina MiSeq platform in an overlapping $2 \times 300 \mathrm{bp}$ configuration to obtain a minimum throughput of 20,000 sequences (reads) per sample.

\subsection{Sequence Analysis and Taxonomical Assignation}

The ITS amplicons were processed and analyzed by adapting previously described protocols [23,24]. Briefly, reads were overlapped by pairs and cleaned out of barcodes. Sequences $<150 \mathrm{bp}$ or with ambiguous assignation were discarded. Valid sequences were grouped using USearch (v. 6.1.544) with $4 \%$ of divergence in order to remove chimeras and singletons [25,26]. Finally, sequences were filtered with a minimum quality of 30 (q30) with Mothur v. 1.22.2 [27]. Taxonomical assignation was done using the software Quantitative Insights Into Microbial Ecology, QIIME v. 1.9.1 [28]. Operational Taxonomical Units (OTUs) were identified at 95\% identity against Unite Community ITS database (v. 7.2) [29] with USearch v. 6.1.544 [25,26] using default parameters in QIIME. The OTUs with mitochondrial or chloroplast assignation were removed. The OTUs identification numbers, abundance and taxonomy retrieved from UNITE database for all samples are specified in Supplementary Table S1. All ITS sequence data used in this study were deposited in the Sequence Read Archive (SRA) of the National Center for Biotechnology Information (NCBI) under the BioProject accession number PRJNA601147.

\subsection{Alpha Diversity of Samples}

To perform alpha-diversity analyses, each sample was randomly subsampled (without replacement) using the alpha_rarefaction.py script found in QIIME 100 to generate Shannon and Chao1 indices along with the observed number of OTUs at different sampling depths. Rarefaction curves for each of these metrics were obtained by serial subsampling (in increments of 5509 sequences and 10 iterations per increment) to a standardized depth of 55,000 sequences per sample.

\subsection{Principal Components (PCA)}

A Principal Components Analysis (PCA) was performed using R package vegan v. 2.5.6 [30] over $R$ v. 3.6.0 to examine compositional changes in the fungal community between wine unfermented must samples from 2016 and 2017 seasons. Our data consisted of 182 OTUs by a 12 sample matrix.

\subsection{Co-Occurrence Networks Based on Co-Presence and Mutual Exclusion}

To examine changes in the composition of the fungal community, we generated co-occurrence networks as described in Mandakovic et al., 2018 [31]. Briefly, significant co-presences or mutual exclusions across the samples were identified by the CoNet method [32] using a multiple ensemble correlation. Four similarity measures were calculated: Bray Curtis and Kullback-Leibler non-parametric dissimilarity indices; Pearson and Spearman rank correlations. In order to cope with the requirements of the CoNet method [32], a distribution of all pairwise scores between OTUs was computed for all $\mathrm{M}$ and EF samples $(n=12)$ and OTUs that occurred in less than eight samples and with relative abundances below $0.01 \%$ were discarded. For each edge, 1000 renormalized permutations and bootstrap scores 
were generated according to [33]. Cytoscape [34] was used for the graphic representation of the networks. OTUs were included as nodes in the networks.

\subsection{Yeast Isolation and Molecular Identification}

To obtain yeast isolates, $100 \mu \mathrm{L}$ of unfermented must samples from years 2016 and 2017 were diluted $1 / 10,1 / 100$, and 1/1000 in a sterile physiological solution and plated in triplicates in WL Nutrient Agar medium supplemented with chloramphenicol $(100 \mathrm{mg} / \mathrm{L})$ for yeasts over bacteria growth selection. All plates were incubated at $25^{\circ} \mathrm{C}$ for 10 days. Thirty-three colonies with different morphologies were selected and isolated from the plates. Pure isolates were cultured in YPD medium (yeast extract $10 \mathrm{~g} / \mathrm{L}$, peptone $20 \mathrm{~g} / \mathrm{L}$, dextrose $20 \mathrm{~g} / \mathrm{L}$ ) at $25^{\circ} \mathrm{C}$ and stored at $-80{ }^{\circ} \mathrm{C}$ after addition of glycerol $(30 \% \mathrm{v} / \mathrm{v})$. To extract yeast DNA, isolated colonies were resuspended in sterile PBS and boiled for $15 \mathrm{~min}$ at $95^{\circ} \mathrm{C}$. Samples were centrifuged at 13,000 rpm for $5 \mathrm{~min}$ and the supernatant was used as DNA template. For ITS PCR amplifications, primers ITS-1 (5'-TCCGTAGGTGAACCTGCGG-3') and ITS-4 (5'-TCCTCCGCTTATTGATATGC-3') were used. ITS PCR amplifications were carried out in $25 \mu \mathrm{L}$ volumes containing $200 \mathrm{ng}(\sim 2 \mu \mathrm{L})$ of fungal DNA, $12.5 \mu \mathrm{L}$ of MixGoTaq Green (Promega, Madison, WI, USA), $8.5 \mu \mathrm{L}$ of nuclease-free water, and $1 \mu \mathrm{L}$ of each primer $(10 \mathrm{mM})$. The PCR amplification was performed in MJ Research, Inc. Thermal cycling controller with the following protocol: $5 \mathrm{~min}$ at $95^{\circ} \mathrm{C}, 35$ cycles of $95^{\circ} \mathrm{C}$ for $30 \mathrm{~s}, 60^{\circ} \mathrm{C}$ for $30 \mathrm{~s}$, and $72{ }^{\circ} \mathrm{C}$ for $60 \mathrm{~s}$, and a final extension at $72{ }^{\circ} \mathrm{C}$ for $10 \mathrm{~min}$. PCR products were visualized in $2 \%(w / v)$ agarose gel electrophoresis in Tris-acetate-EDTA (TAE) buffer $(1 \times)$ and stained with ethidium bromide. PCR products were purified and sequenced in Macrogen Korea, while the identification of genus and species was based on the best sequence match obtained using NCBI database. Isolate sequences obtained in this study are available in NCBI database (see Supplementary Table S5). In addition, the reads from the ITS1/2 amplicon sequencing of yeast population were mapped to the ITS1/4 sequences of isolates using BLASTN.

\subsection{Microcultivation Assay}

The microcultivation assay was performed as previously described [35]. Briefly, strains were precultivated in $200 \mu \mathrm{L}$ of YNB medium ( $0.67 \%$ yeast nitrogen base, $2 \%$ glucose) for $48 \mathrm{~h}$ at $25^{\circ} \mathrm{C}$. Subsequently, strains were inoculated to an optical density (OD) of 0.03-0.1 (wavelength $620 \mathrm{~nm}$ ) in $200 \mu \mathrm{L}$ of media (either YNB or synthetic wine must, SWM) and incubated without agitation at $25^{\circ} \mathrm{C}$ in a TECAN Sunrise absorbance microplate reader. OD was measured every 30 min using a $620 \mathrm{~nm}$ filter. Each experiment was performed in triplicates. Maximum growth rate, lag time, and OD max for each strain were calculated using GrowthRates software with default parameters [36]. Relative growth was estimated compared to YNB (yeast nitrogen base + glucose $2 \%$ ) and a z-score regression was performed to normalize values within each condition. In all cases, $p$ values were obtained using a one-way ANOVA.

\subsection{Fermentations and HPLC Analysis}

Fermentations were carried out as previously described [37]. Briefly, each strain was fermented in quadruplicate in SWM MS300 (Synthetic must contain $300 \mathrm{mgN} / \mathrm{L}$ ) and prepared according to Rossignol et al [38]. SWM was supplemented with a final concentration of $300 \mathrm{mgN} / \mathrm{L}$ of assimilable nitrogen (YAN) corresponding to $120 \mathrm{mgN} / \mathrm{L}$ of ammonium and $180 \mathrm{mgN} / \mathrm{L}$ of a mixture of 19 amino acids $(612.6 \mathrm{mg} / \mathrm{L}$ L-proline, $503.5 \mathrm{mg} / \mathrm{L}$ L-glutamine, $503.5 \mathrm{mg} / \mathrm{L} \mathrm{L}$-arginine monohydrochloride, $179.3 \mathrm{mg} / \mathrm{L}$ L-tryptophan, $145.3 \mathrm{mg} / \mathrm{L}$ L-alanine, $120.4 \mathrm{mg} / \mathrm{L} \mathrm{L}$-glutamic acid, $78.5 \mathrm{mg} / \mathrm{L} \mathrm{L}$-serine, $75.92 \mathrm{mg} / \mathrm{L} \mathrm{L}$-threonine, $48.4 \mathrm{mg} / \mathrm{L}$ L-leucine, $44.5 \mathrm{mg} / \mathrm{L} \mathrm{L}$-aspartic acid, $44.5 \mathrm{mg} / \mathrm{L}$ L-valine, $37.9 \mathrm{mg} / \mathrm{L} \mathrm{L}$-phenylalanine, 32.7 $\mathrm{mg} / \mathrm{L} \mathrm{L}$-isoleucine, $50.0 \mathrm{mg} / \mathrm{L} \mathrm{L}$-histidine monohydrochloride monohydrate, $31.4 \mathrm{mg} / \mathrm{L} \mathrm{L}$-methionine, $18.3 \mathrm{mg} / \mathrm{L}$ L-tyrosine, $18.3 \mathrm{mg} / \mathrm{L}$ L-glycine, $17.0 \mathrm{mg} / \mathrm{L}$ L-lysine monohydrocloride, and $13.1 \mathrm{mg} / \mathrm{L}$ L-cysteine). The strains were initially grown under constant agitation in $10 \mathrm{~mL}$ of SWM for $48 \mathrm{~h}$ at $25^{\circ} \mathrm{C}$. Next, $1 \times 10^{6}$ cells $/ \mathrm{mL}$ were inoculated into $10 \mathrm{~mL}$ of SWM (in $15 \mathrm{~mL}$ conical tubes) and incubated at $25^{\circ} \mathrm{C}$. Fermentations were weighed every day to calculate the $\mathrm{CO}_{2}$ output until the daily 
$\mathrm{CO}_{2}$ loss represented less than $10 \%$ of the accumulated $\mathrm{CO}_{2}$ loss. Similarly, fermentations in $50 \mathrm{~mL}$ were carried out in $250 \mathrm{~mL}$ flasks with constant agitation using a magnetic stirrer at $25^{\circ} \mathrm{C}$ and $650 \mathrm{rpm}$ (DLAB Scientific, Beijing, China). Final fermented SWMs, were centrifuged at $9000 \times g$ for $10 \mathrm{~min}$ and the supernatant was collected. $20 \mu \mathrm{L}$ were injected in a Shimadzu Prominence HPLC equipment (Shimadzu, Tokyo, Japan) using a Bio-Rad HPX-87H column to estimate final concentrations of: glucose, fructose, acetic acid, glycerol, and ethanol [39]. Glycerol and ethanol yields were estimated as previously described [37].

\subsection{Competition Experiments}

Relative fitness in fermentation conditions was estimated using a competition assay. For this, strains were initially grown under constant agitation in $10 \mathrm{~mL}$ of SWM for $48 \mathrm{~h}$ at $25^{\circ} \mathrm{C}$. Subsequently, $1 \times 10^{6}$ cells/mL or $1 \times 10^{4}$ of EC1118 (hsp12::GFP-KanMX, [40] and $1 \times 10^{6}$ cells $/ \mathrm{mL}$ of the competing strain (the four fast fermenters: Saccharomyces uvarum Torulaspora delbrueckii, M. pulcherrima, and Zygotorulaspora florentina) were inoculated into $125 \mathrm{~mL}$ of SWM and kept under constant agitation (200 rpm) at $25^{\circ} \mathrm{C}$ during five days. Chilean native S. cerevisiae isolates were excluded from competition experiments because their potential in wine fermentation has been previously reported [41]. Relative fitness was estimated at $0,24,48,72$, and $96 \mathrm{~h}$ post-inoculation quantifying the fraction of G418 resistant colonies versus the total number of colonies on YPD-Agar plate (yeast extract $1 \%$, peptone $2 \%$, glucose $2 \%$, and agar $1.5 \%$ ).

\section{Results}

\subsection{Fungal Composition in Unfermented and Fermented Musts from Two Consecutive Seasons} of Wine Production

We used two types of samples from the wine making process: the unfermented must (M) and the fermented must (wine just before bottling at the end of fermentation using a commercial Saccharomyces cerevisiae strain; hereinafter EF). Analysis of the raw sequence data yielded 1,431,165 reads after quality trimming. Taking together both seasons, we identified 182 OTUs (Supplementary Table S1), using a 95\% identity threshold against the Unite Community ITS database and with $\geq 0.01 \%$ of relative abundance in at least two of the three replicates of $\mathrm{M}$ or EF at each season. These OTUs were affiliated to 133 species belonging to 52 genera (Supplementary Table S1) and mainly ( $>99 \%$ of the total relative abundance per sample) to the Ascomycota division. Additionally, microbiome data indicated that $11.5 \%$ of the OTUs belonged to unidentified genera (21 out of 182 total OTUs), revealing an important proportion of putative new fungal species in this environment. The most abundant genus in unfermented and fermented musts was Saccharomyces (average 53 and 91\%, respectively, Figure 1A), mainly due to the contribution of Saccharomyces uvarum (42.7\%) and Saccharomyces cerevisiae ( $80 \%)$.

In the first season, $\mathrm{M}$ and $\mathrm{EF}$ were both represented by 25 common or core genera (Supplementary Table S1), while 13 were exclusive for M (Erysiphe, Rhodosporidiobolus, Kabatiella, Trichoderma, Talaromyces, Leptoxyphium, Malassezia, Hansfordia, Seimatosporium, Sarocladium, Filobasidium, Holtermanniella, and Rhodotorula) and only four very low abundant genera (with less than $0.01 \%$ of relative abundance) were exclusive for EF (Zygoascus, Didymosphaeria, Cadophora, and Curvibasidium) (Supplementary Table S1). On the other hand, in the second season, M and EF had 23 core genera affiliated, where $\mathrm{M}$ had 16 exclusive genera, and EF only had a single low abundant exclusive genus (Schwanniomyces, $0.002 \%$ of relative abundance) (Supplementary Table S1).

Regarding solely M samples, from the 48 total genera present in this sample, 29 were core between both seasons; meanwhile, nine were exclusive of the first season, and 10 of the second season (Supplementary Table S1). Differences between seasons were also reflected in a significant difference $(p<0.05$, ANOVA) in richness among the unfermented samples (Supplementary Table S2, Chao-1 index), and in a Principal Component Analysis (PCA; Supplementary Figure S1), in which PC1 explained $80 \%$ of the variation in community composition (Supplementary Figure S1). Between 
seasons, two genera, Hanseniaspora and Torulaspora, were highly different in their relative abundances among the M samples. Specifically, Hanseniaspora accounted for $36.2 \%$ of the relative abundance present in year 2016, but only for 3.1\% in year 2017, whereas Torulaspora accounted for $19.3 \%$ of the relative abundance in 2016 and only for 4.8\% in year 2017 (Supplementary Table S1, Figure 1A). Concerning the physicochemical parameters of the unfermented musts, they were similar among seasons (Supplementary Table S3).

EF samples showed a different pattern compared to $M$ samples. While $60.6 \%$ of the genera from these samples were core $(n=20)$, only nine and four genera were exclusive to the first and second seasons, respectively (Supplementary Table S1). This data was also reflected in the absence of significant difference of richness values between seasons for EF samples (Supplementary Table S2, Chao-1 index). Additionally, in both seasons, the EF Shannon value was significantly lower than in $\mathrm{M}(p<0.05$, ANOVA, Supplementary Table S2), suggesting that a higher diversity was present in unfermented over fermented musts.

A

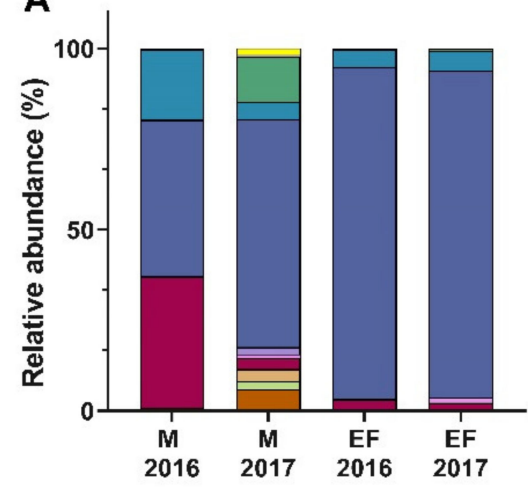

B

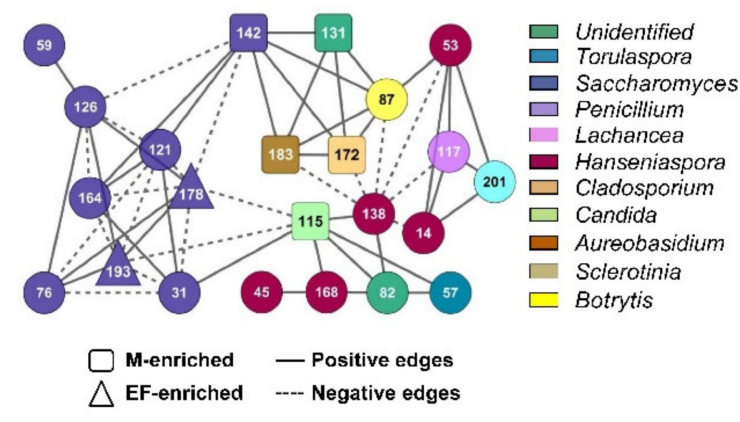

Figure 1. Fungal assemblages of unfermented and end of fermentation stages. (A) Average relative abundances of genera from unfermented $(\mathrm{M})$ and end of fermentation $(\mathrm{EF})$ samples $(>0.1 \%$ of relative abundance in at least one sample) based on massive sequencing of Operational Taxonomical Units (OTUs) in triplicates. (B) Fungal interaction network of $\mathrm{M}$ and EF samples from both seasons.

The putative ecological interactions among different fungal species within $\mathrm{M}$ and EF communities were examined by a microbial interaction network analysis (Figure 1B). For the purpose of having an appropriate number of samples to cope with the requirements of the CoNet method for network analysis [32], the six $\mathrm{M}$ and six EF samples from the two consecutive seasons of production were used. Network properties such as the number of links of the node (degree) and interaction type (nodes with positive and negative links, representing co-presences and mutual exclusions, respectively) were listed (Supplementary Table S4). From a total of 182 OTUs identified, 23 OTUs belonging to nine genera were engaged into the network, which showed a higher proportion of positive $(n=76)$ than negative interactions $(n=40)$. It is important to mention that positive or negative interactions within fungal communities can represent ecological interactions (co-presences or mutual exclusions), or simply indicate that they are responding in the same way to a change in environmental conditions [42,43], thus a functional analysis of isolates is needed to interpret these interaction patterns. Interestingly, Saccharomyces genus was represented by the greatest number of OTUs $(n=9)$ within the network, exhibiting the highest degree values. Moreover, three OTUs belonging to this genus were enriched ( $>20$ folds) at M or EF or samples (Supplementary Table S4).

\subsection{Identification of Yeast Species of Unfermented Grape Musts}

A total of 33 distinct colonies were isolated from unfermented musts, which were differentiated based on colony color and morphology on WL nutrient agar. The ITS1 and ITS4 regions of the 33 isolated yeasts were then sequenced for species identification. Out of this, we identified 
individuals belonging to six genera and nine species as follows: Zygotorulaspora florentina, Torulaspora delbrueckii, Saccharomyces ovarum, Saccharomyces cerevisiae, Starmerella bacillaris, Metschnikowia pulcherrima, Pichia fermentans, Pichia membranifaciens, and Pichia manshurica. Supplementary Table S5 provides an overview of the isolated representative strains, their ITS region fragment sizes, and their GenBank accession numbers.

When reads from HTS were mapped to the ITS sequences of isolates, the relative abundances of the isolated strains in M and EF samples were calculated for years 2016 and 2017 (Figure 2). Eight out of nine isolates were present in $\mathrm{M}$ and $\mathrm{EF}$ at both sampling seasons, but with different relative abundances. At year 2016, T. delbrueckii represented $19.3 \%$ and $4.8 \%$ of the population in M and EF, respectively, while at year 2017, it was present at similar levels in both samples (4.9\% in M and 5.3\% in EF). M. pulcherrima, P. fermentans, P. membranifaciens, Z. florentina, and S. bacillaris were found at very low abundances at both sampling seasons, whereas $S$. uvarum was highly abundant in M samples (31.4\% and $54.1 \%$ for years 2016 and 2017, respectively) and then decreased in EF samples (3.5\% in 2016 and 1.7\% in 2017). In contrast, S. cerevisiae increased in abundance to become the dominant genus in the EF samples $(78.6 \%$ in 2016 and $81.5 \%$ in 2017). One of the isolates, P. manshurica, was the only species gained in culture but absent from the microbiome sequencing. This may be due to a low efficiency of DNA isolation from cells of this species and/or to a very low abundance of this yeast in the unfermented musts samples, which could have prevented its detection by the sequencing strategy.

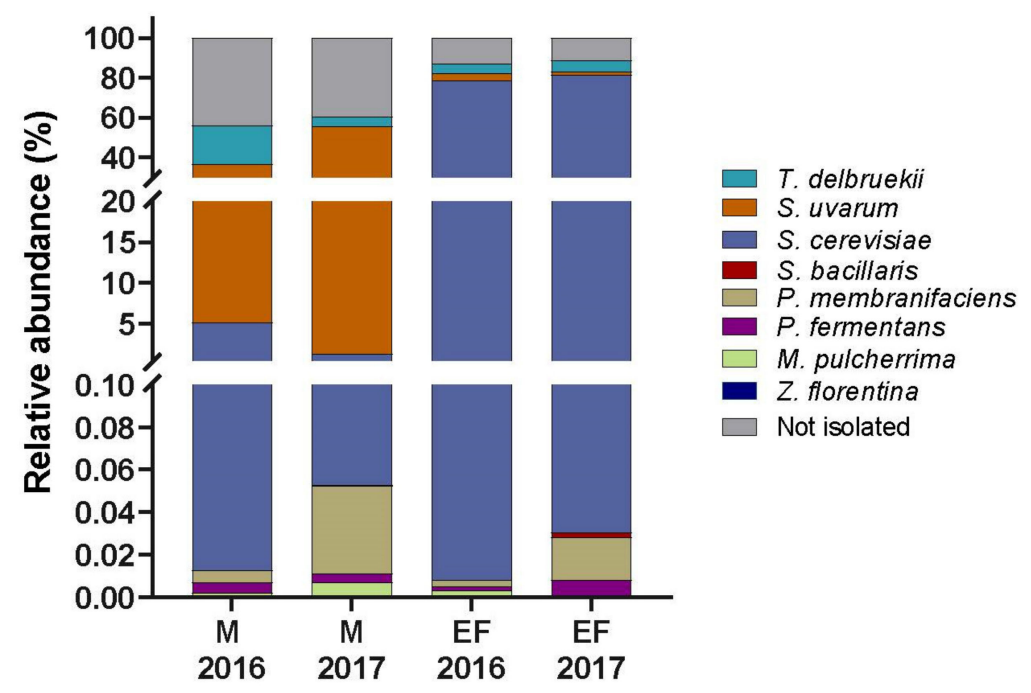

Figure 2. Relative abundance of isolates in unfermented and end of fermentation stages. Reads from high-throughput sequencing (HTS) were mapped to the internal transcribed spacer (ITS) sequences of isolates to calculate the average relative abundances of isolated species from unfermented (M) and end of fermentation (EF) samples. In grey, relative abundance of the remaining species that were not isolated but were massive sequenced.

\subsection{Fermentation Performance of Natural yYeasts Isolates on Synthetic Wine Must MS300}

In order to determine the ability of isolated yeast to generate biomass under wine must fermentation and laboratory growth conditions, a high-throughput microcultivation assay was performed under synthetic wine must MS300 (SWM) and YNB media, respectively, and from this we determined: lag phase, maximum growth rate ( $\mu$ max), and maximum OD (OD max) (Supplementary Table S6). Interestingly, some non-conventional species such as Torulaspora delbrueckii showed high $\mu$ max and OD max under SWM ( $p$ value $<0.05$, ANOVA) like Saccharomyces strains, suggesting a high degree of adaptation to this hostile environment (Figure 3A). For Pichia species a low $\mu$ max was observed under SWM, except for Pichia fermentans which showed a greater $\mu$ max compared to the other three Pichia species analyzed. On the contrary, under laboratory conditions (YNB + 2\% Glucose), Pichia strains showed a higher $\mu$ max and OD max relative to SWM (Supplementary Table S6). Subsequently, 
we evaluated a series of conditions normally found in wine: the hyperosmotic stress response in glucose 25\% and fructose 25\%, together with ethanol resistance (ethanol 9\%) and estimated their relative growth rate ( $\mu \max$ ) to YNB supplemented with glucose $2 \%$ (Figure $3 \mathrm{~B}$ ). Interestingly, two Pichia strains (P. membranifaciens and P. manshurica) clustered separately from the other strains and were tolerant to hyperosmotic and ethanol 9\% stress, where hyperosmotic stress could explain their prevalence in non-fermented wine must (high hyperosmotic media). On the other hand, T. delbrueckii clustered close to Saccharomyces strains, exhibiting a high relative growth level under most conditions, except for fructose $25 \%$ where lower $\mu$ max were observed (Supplementary Table S6). M. pulcherrima clustered together with $P$. fermentans, mostly due to a low hyperosmotic stress, ethanol tolerance and fructose uptake. Saccharomyces strains showed the highest ethanol and SWM $\mu$ max, clustering together for all estimated parameters (Supplementary Figure S2). These results demonstrate the dominance of Saccharomyces strains across wine-related phenotypes; however, it also highlights that other non-conventional yeasts are able to tolerate high sugar and/or ethanol levels.

A

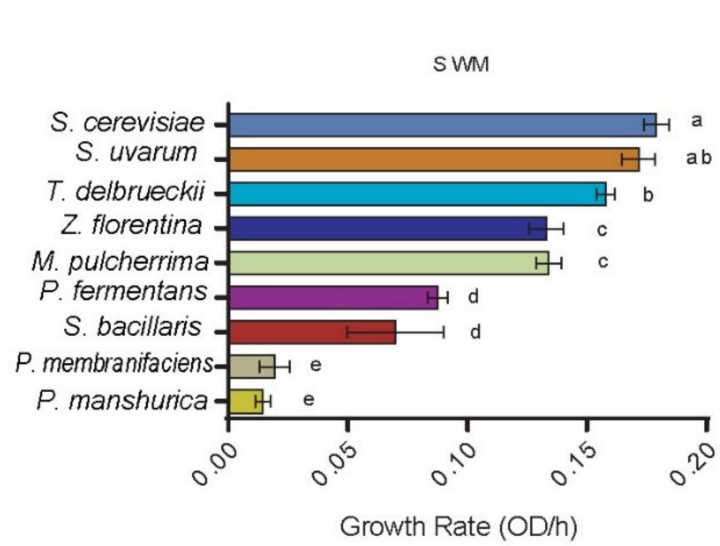

B

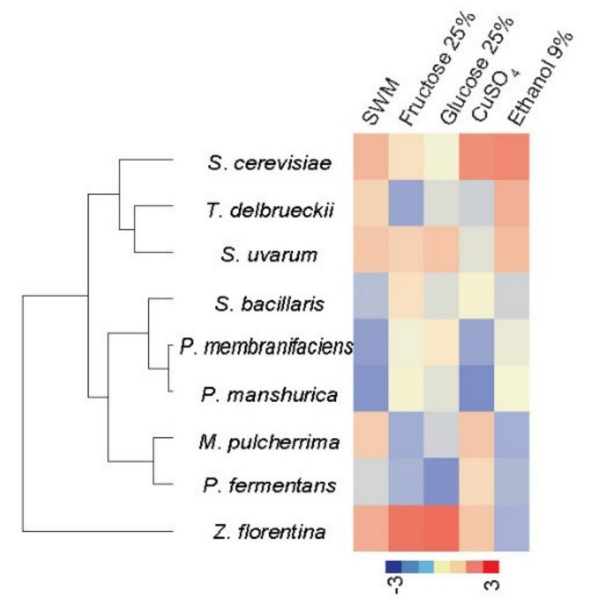

Figure 3. Wine-related phenotypes in natural strains. (A) Maximum growth rate ( $\mu \mathrm{max}, \mathrm{OD} / \mathrm{h}$ ) in synthetic wine must (SWM), a,b,c,d,e depict significant differences ( $p$-value $<0.05$, ANOVA).

(B) Heatmap from all phenotypes evaluated under micro-cultivation conditions.

Since most strains were able to grow under microcultivation conditions, we next evaluated their fermentation capacity under micro-fermentations in SWM. For this, $1 \times 10^{6}$ cells $/ \mathrm{mL}$ of each strain were inoculated in $10 \mathrm{~mL}$ and micro-fermentations were carried out at $25^{\circ} \mathrm{C}$ for $\sim 21$ days. Subsequently, we estimated the $\mathrm{CO}_{2}$ loss for the fermentation period representing a fermentation efficiency trait. Overall, we distinguished two groups clearly differentiated based on their total $\mathrm{CO}_{2}$ release: fast fermenters (FF) and slow fermenters (SF) (Figure 4A). Among the FF species, we observed the two Saccharomyces species: S. cerevisiae and S. uvarum followed by three non-conventional species: T. delbrueckii, M. pulcherrima, and Z. florentina. The SF group contained four non-conventional species: T. delbrueckii, M. pulcherrima, and Z. florentina. The SF group contained four non-Saccharomyces species: S. bacillaris and the three Pichia species, P. fermentans, P. membranifaciens, and P. manshurica. In agreement with the results obtained under microcultivation conditions, the Pichia sp. showed almost none or low $\mathrm{CO}_{2}$ loss levels (Figure 4A), demonstrating their incapacity to colonize the wine fermentation environment. 


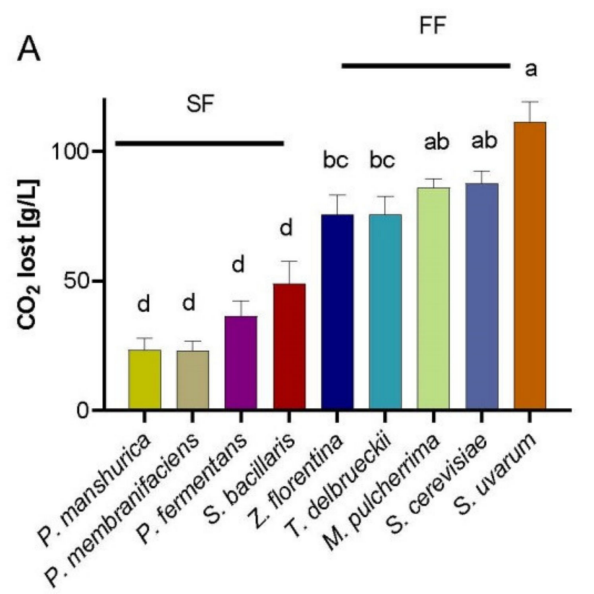

B

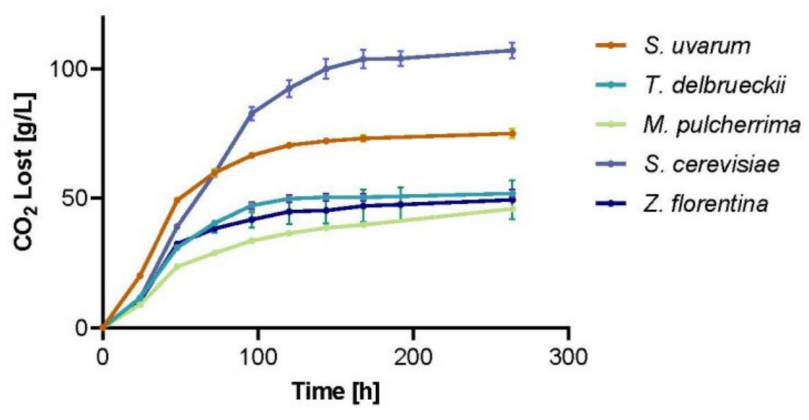

Figure 4. Fermentative capacity of natural strains. (A) Total $\mathrm{CO}_{2}$ lost in all strains in $10 \mathrm{~mL}$ fermentations (SF, Slow Fermenters and FF, Fast Fermenters), a,b,c,d,e depict significant differences (p-value < 0.05, ANOVA). (B) FF under $50 \mathrm{~mL}$ fermentations with agitation. EC1118 was used as the commercial S. cerevisiae control.

To evaluate the whole fermentative potential of each individual under SWM, we measured fermentation performance of the FF species under a greater SWM volume $(50 \mathrm{~mL})$ in constant agitation and included the commercial, and widely characterized, S. cerevisiae strain (EC1118) as comparison control (the isolated S. cerevisiae strain was excluded from our analysis). Interestingly, S. uvarum exhibited greater $\mathrm{CO}_{2}$ loss levels than any of the other species, except for the renowned commercial wine yeast (Figure 4B), demonstrating its potential as top fermenter. On the other hand, the three non-conventional species showed lower $\mathrm{CO}_{2}$ loss levels compared to the Saccharomyces species, exhibiting a lower fermentation performance (Figure 4B). These results demonstrate the greater fermentation capacity of native Saccharomyces strains compared to other non-conventional isolates. Still, the latest are able to efficiently ferment the SWM and represent new candidate strains for wine fermentation.

\subsection{Determination of Organic Acid Composition in Synthetic Wine}

Fast fermenters represent potential new starter strains for a controlled fermentation process [3]. In order to determine their ability to consume sugar sources provided in the SWM and the resultant production of other organic acids, we estimated through HPLC the concentrations of important metabolites such as: glucose, fructose, trehalose, acetic acid, ethanol, and glycerol at the end of the fermentation (Supplementary Table S7). As expected, all FF species consumed at least $~ 50 \%$ of the glucose provided, in contrast to fructose where some strains consumed less than $40 \%$ of the provided amount (Figure 5A). The commercial isolate consumed all the available sugars ( $250.9 \mathrm{~g} / \mathrm{L})$, while S. uvarum consumed only $67 \%$ of fructose and glucose.

The production of glycerol and ethanol represent attractive metabolites during wine fermentation, while acetic acid represents an undesirable trait. Interestingly, for M. pulcherrima the consumption of glucose and fructose translated into the highest glycerol yields among all strains ( $p$-value $<0.05$, ANOVA), with over 2.23 times more than the one obtained by EC1118. In contrast, ethanol levels for M. pulcherrima were significantly lower compared to Saccharomyces species ( $p$-value $<0.05$, ANOVA, Figure 5B); however, both species exhibited similar yield values ( $p$-value $>0.05$, ANOVA, Figure 5C), and did not reach commercial standards (Supplementary Table S7). T. delbrueckii and Z. Florentina also showed greater glycerol levels compared to ethanol, yet in all cases glycerol levels were lower compared to EC1118 and S. uvarum (Figure 5B). Interestingly, with both species higher yield values were observed when compared to EC1118 (Figure 5C and Supplementary Table S7). On the contrary, acetic acid levels were significantly high in the two Saccharomyces species, while the non-Saccharomyces 
showed less than $1 \mathrm{~g} / \mathrm{L}$ of this undesired compound (Figure 5D). In addition, the $S$. uvarum isolate also exhibited high production levels of ethanol and glycerol; however, this strain showed significantly lower levels of acetic acid production compared to the commercial strain under our fermentative conditions. These results demonstrate the potential of the native S. uvarum and the non-conventional yeast M. pulcherrima for their utilization as starter culture in wine fermentation. However, further assays could be performed in greater volumes under non-synthetic wine must conditions to validate these results.
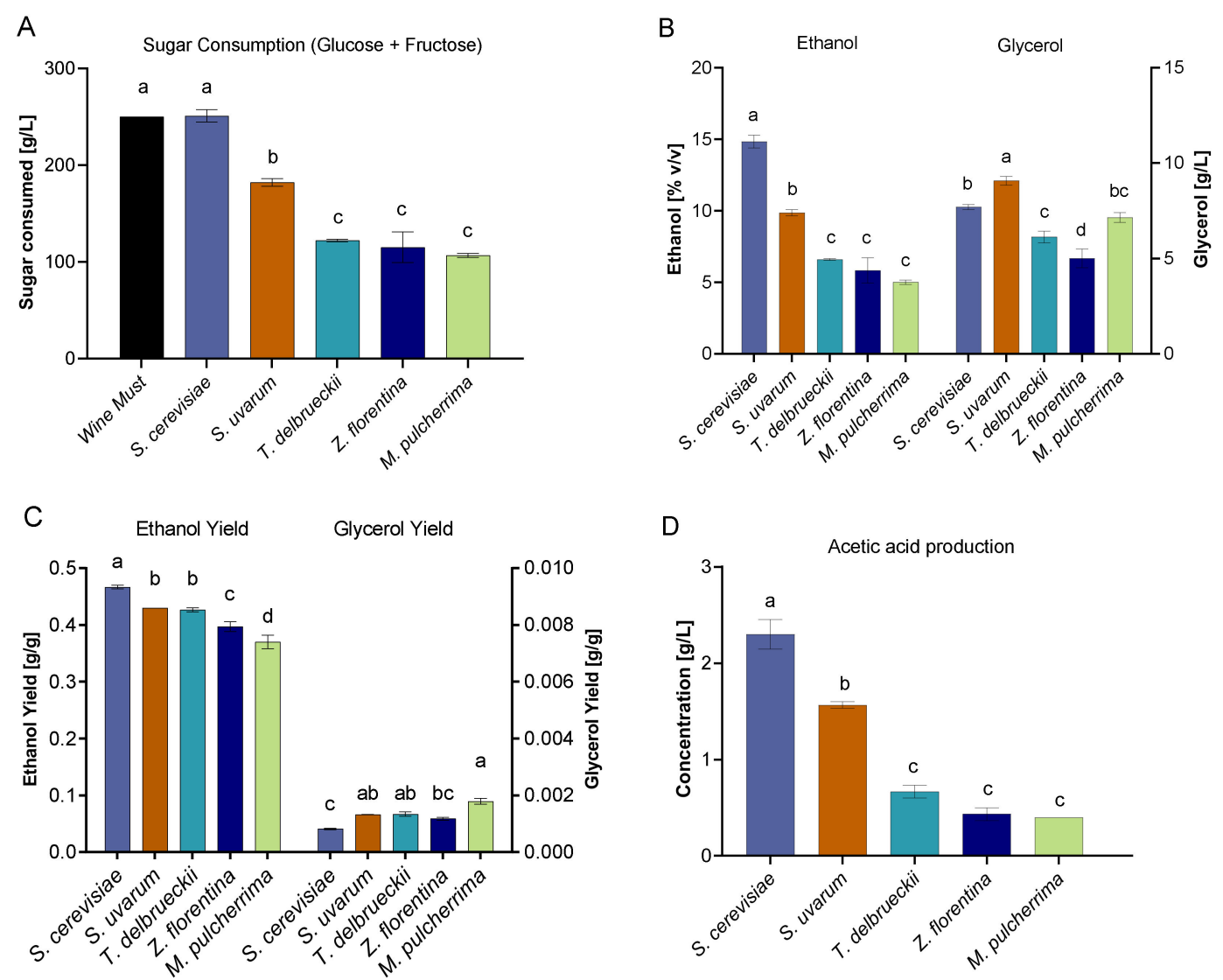

Figure 5. HPLC results from fermentation with Saccharomyces and non-Saccharomyces strains. (A). Sugar consumption, (B) Ethanol and Glycerol production, (C) Ethanol and Glycerol yields, and (D) Acetic acid production.

\subsection{Competitive Performance of Native Strains in Co-Cultures Systems}

In order to determine the competitive capacity against $S$. cerevisiae of the non-Saccharomyces and S. uvarum strains, we co-inoculated in SWM each strain together with a geneticin resistant strain derived from the S. cerevisiae EC1118 background [40] and determined their abundance relative to the commercial strain during $100 \mathrm{~h}$. In all cases, the commercial S. cerevisiae strain out-competed the others after $48 \mathrm{~h}$, except for M. pulcherrima, for which viable cells were found even after $100 \mathrm{~h}$ of co-cultivation (Figure 6A). Interestingly, S. uvarum was rapidly overtaken by EC1118, representing the strain with the lowest comparative fitness. The T. delbrueckii and Z. florentina strains showed similar competitive profiles, being overtaken by EC1118 after $72 \mathrm{~h}$. Subsequently, to determine whether the isolated strains were able to dominate the fermentation environment when inoculated in excess compared to EC1118, the co-cultivation assay was modified and $1 \times 10^{6}$ and $1 \times 10^{4}$ cells $/ \mathrm{mL}$ of non-Saccharomyces (and S. uvarum) were inoculated together with EC1118, respectively. In this case, M. pulcherrima and S. uvarum were the sole species able to maintain dominance over the culture and were not dominated by EC1118 (Figure 6B). Altogether, these results suggest that our strain of M. pulcherrima represents the 
sole non-Saccharomyces native strain that could co-exist for a longer period in co-cultivation with a commercial S. cerevisiae strain under wine fermentative conditions.

\section{A}
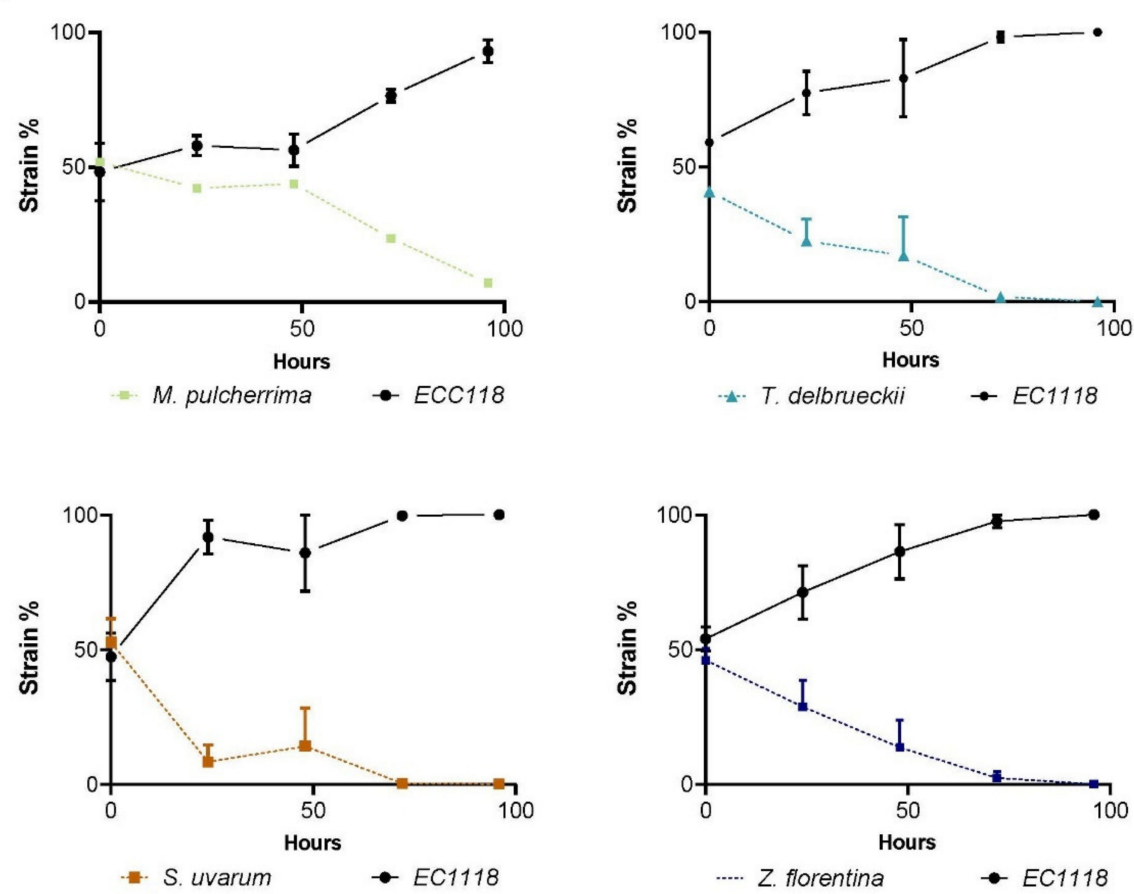

B
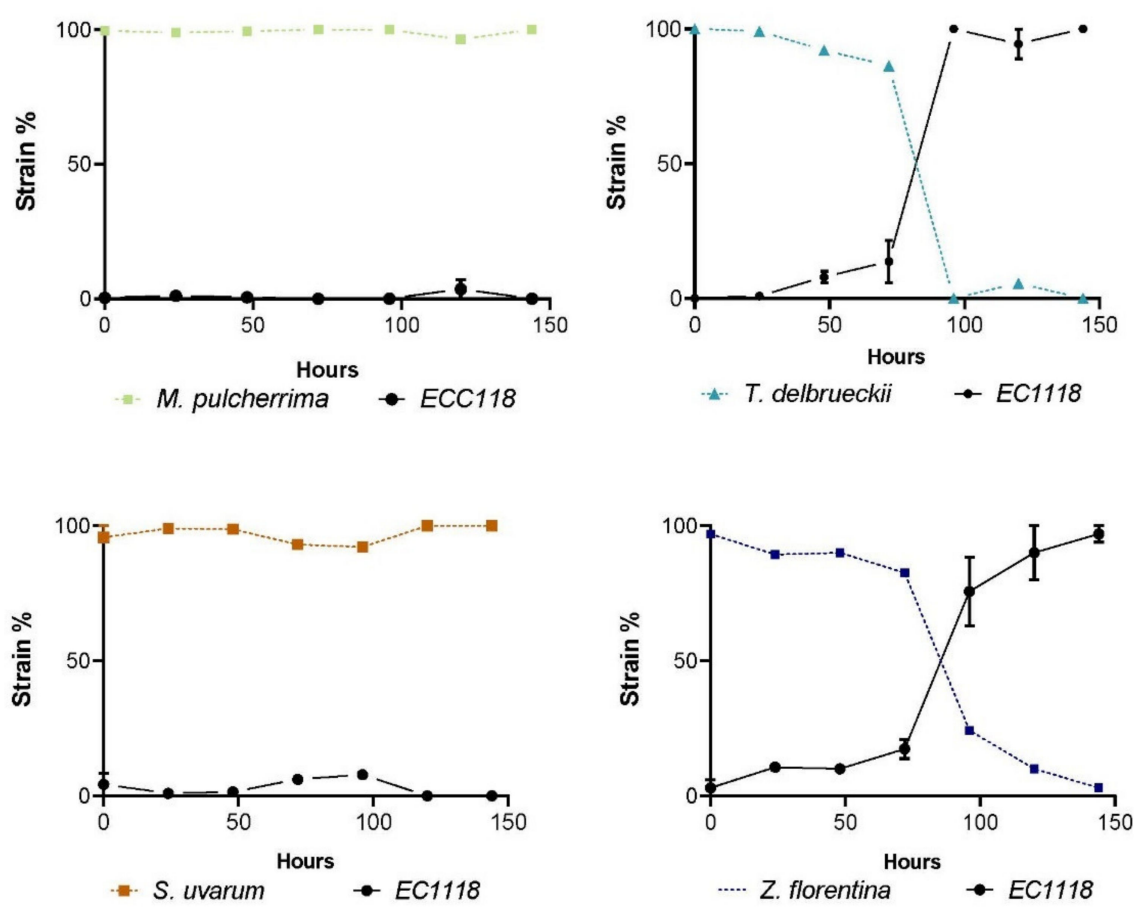

Figure 6. Competition experiment between commercial strains versus natural isolates. The relative percentage of the reporter commercial strain EC1118 (black line) in competition with each of the other FF strains inoculated in (A) equal proportions and (B) 1:100 proportion. 


\section{Discussion}

In this work, we monitored, during two seasons, M- and EF-associated fungal assemblages from the variety of Vitis vinifera L var. Sauvignon blanc in a commercial winery. Taking into account that the diversity of yeasts during grape must fermentation [21] or associated with grape berries in different Chilean valleys [20] have been described using mainly cultivation techniques and/or qPCR-based assays, here we performed a HTS approach based on the ITS-sequencing of all the eukaryotic microorganisms present in the unfermented and fermented musts in consecutive seasons. Our aims were: 1) to compare $\mathrm{M}$ and EF community structures by identifying the core community of indigenous wine yeasts and to determine their putative ecological interactions, and 2) to isolate and characterize cultivable native yeasts as potential new starters for winery industry.

Although only two seasons were analyzed, we observed that $\mathrm{M}$ and EF samples shared a common fungal community (core microbiome, 56 OTUs) dominated by species belonging to Ascomycota phylum, while species of Basidiomycota represented less than $0.2 \%$ of the population in all samples (Supplementary Table S1), similar to the results that have been reported for different red and white wine grapes [44,45]. Taxonomic analysis also showed that all species of the Saccharomyces genus could be recovered consistently in the two sequential seasons in both $\mathrm{M}$ and EF samples. The exclusive OTUs found in the M (non-core microbiome) most probably failed to grow up under stress conditions generated during the fermentation process [46].

Additionally, we observed significant seasonal microbial variability in $M$ samples (Supplementary Table S2), such as in genera Hanseniaspora and Torulaspora, which showed notable differences in relative abundances among seasons (Supplementary Table S1, Figure 1A). This variability could not be explained by the physicochemical parameters measured in these samples, but rather it might be explained by other environmental factors that we did not evaluate and could have affected the microbial community prior to the harvest, such as temperature, relative humidity and rainfall, among others $[8,15]$. We noticed that from the 48 total genera present in $\mathrm{M}$ samples, the exclusive ones from the first and second seasons of the unfermented musts were represented by few reads, adding only 0.004 and $0.012 \%$ of relative abundance, respectively (Supplementary Table S1). These results indicate that the differential richness observed among must samples in consecutive years was mainly attributable to rare species that most likely own differential tolerances towards the parameters measured, and thus these factors could have a role controlling the fungal community structure.

Regarding the presence of low abundance taxa, Mendoza et al. (2017) also reported that unfermented samples were characterized by many species represented by very few reads in the ITS sequencing data, whereas yeast communities of fermented samples comprised few species represented by many reads. Accordingly, by the end of fermentation, we observed that the yeast community was practically the same in both years, with no significant differences in Chao-1 nor in Shannon indices (Supplementary Table S2), being Saccharomyces consistently the most abundant fungal genus with over $90 \%$ of the relative abundance (Supplementary Table S1). Interestingly, nine OTUs belonging to the Saccharomyces genus were found, including S. mikatae and S. kudriavzevii, which may represent the first evidence of the presence of these species in wine-environments in South America [47]. These results demonstrate the potential of the HTS approach to effectively capture the diversity of yeast associated to wine musts and suggest the presence of species which have not been previously isolated in the southern hemisphere. However, the interpretation of these results needs to be taken with caution, especially regarding the presence of Saccharomyces species, since low variability has been described for the ITS sequence of species belonging to this genus when compared to other yeasts [48], a feature that might affect species classification. A high abundance of S. cerevisiae is expected during the inoculated fermentation, since this fungal species is predominant throughout the fermentation process due to its resistance to elevated amounts of alcohol. In fact, high alcohol contents were measured in both EF samples, with values of $11.4 \pm 0.2$ and $13.6 \pm 1.3 \% v / v$ for years 2016 and 2017, respectively. In contrast, during the spontaneous fermentation process, Saccharomyces dominates later stages of fermentation, whereas non-conventional yeasts can be predominant in the initial and middle stages [49]. These 
results support the relevance of the fermentation process over yeast selection, and therefore over the structure of the microbial community [14]. Additionally, network analysis enabled us to identify theoretical relationships among the fungal groups [43], showing that members of the Saccharomyces genus are forming a cluster or subnetwork module, as expected when species share specific functions with their phylogenetic group [50]. However, the presence of positive and negative links within this module suggests that despite their phylogenetic similarity, they display a differential response (change of their relative abundance) between $\mathrm{M}$ and $\mathrm{EF}$ states.

Beside Saccharomyces species, nine other yeast genera with relative abundances $>0.1 \%$ in at least one $\mathrm{M}$ and/or EF samples were identified. Among them, species within the genera Torulaspora, Pichia, Lachancea, Candida/Metschnikowia, Aureobasidium, and Hanseniaspora have been described as interesting indigenous yeasts, since they are able to produce desirable compounds and metabolites to improve the wine quality, enzymes with roles in wine production or act as biocontrol agents [12]. In particular, species of Torulaspora and Hanseniaspora, which can positively influence quality parameters of wine [17,51], were detected in both $\mathrm{M}$ and EF samples, but with dissimilar abundances, $>4 \%$ and $1 \%$, respectively. In addition to known wine yeasts, we found, mainly in $\mathrm{M}$ sample and with low abundance, some other genera such as Sclerotinia and Botrytis that comprise necrotrophic plant pathogenic fungi with wide host ranges and environmental persistence $[52,53]$ and the genera Cladosporium and Penicillium encompassing saprophytic molds responsible of organoleptic defects in grapes and wines [54]. All these genera have been previously described as part of the grape and must yeast population [54,55].

In order to select indigenous yeasts to use as potential new starters in the wine industry, we isolated yeasts from the unfermented musts since these samples showed higher richness indices when compared to EF samples. From the nine isolates obtained, only Pichia manshurica was not detected by the sequencing strategy. The enrichment of this yeast in the culture medium may be due to the differential supply of nutritional requirements in the culture medium than in the must, the removal of growth inhibitory components and/or the reduction of competitive interactions within members of the community [56]. Additionally, some abundant species of Saccharomyces and Hanseniaspora genera that were identified in the unfermented musts by the sequencing approach, were not recovered during the isolation process. In this regard, previous reports showed a lack of correlation between the abundance detected by HTS and the possibility of strain isolation [57,58].

As expected, the species of Saccharomyces showed high $\mu$ max and fermentation rates under SWM. In particular, S. cerevisiae strains are extremely efficient during the first $48 \mathrm{~h}$ of fermentation because of their fast sugar and nitrogen source uptake $[59,60]$. These sources are immediately stored in the vacuole and later, during the fermentation process, mobilized out of the vacuole to support growth after depletion of external nitrogen [60]. This ability impedes other yeasts from dominating the culture, making Saccharomyces strains best-adapted species to wine must fermentation. Yet, we characterized the fermentation profile and relative fitness of other species found in grape juice that could represent potential new isolates for either controlled or spontaneous fermentations. The fitness of these strains under microcultivation conditions correlated well with their fermentation profiles. Saccharomyces strains together with T. delbrueckii clustered together in most kinetic parameters estimated (Figure 3B), demonstrating their similar fitness under fermentative conditions.

Three non-conventional yeasts species showed high fermentation rates: T. delbrueckii, M. pulcherrima, and Z. florentina. T. delbrueckii isolates are found naturally in many wine-producing areas, and even recently commercial strains have become available, mostly because of their contribution to the aroma profile [16]. Similarly, M. pulcherrima (also known as Candida pulcherrima) is also available in the market and is well-known for the high esters production, such as the ethyl octanoate (pear-like aroma) [61]. On the other hand, only recently Z. florentina has been considered for wine fermentation, exhibiting high glycerol concentrations and low volatile acids [62]. Here, we obtained lower glycerol levels (and yields) and low acetic acid levels compared to other strains, likely due to the low sugar consumption compared to the other strains. However, glycerol yields were above those obtained in the commercial strain. Indeed, the utilization of Z. florentina in co-cultures has been suggested as a 
favorable strategy to mitigate high volatiles acids production by S. cerevisiae [63]. In this context, our competition experiment demonstrated that non-conventional strains are overtaken when co-cultivated with a commercial $S$. cerevisiae strain, suggesting that co-cultivation could occur only during the first 2-3 days of fermentation and limiting their utilization (Figure 6). Instead, M. pulcherrima demonstrated the greatest fitness compared to the other tested strains when competed against EC1118, being able to survive for a longer period. In this sense, this strain was the sole found to remain after five days in the mixed culture, demonstrating its plausible utilization in co-cultures with $S$. cerevisiae commercial strains. However, further evidence is needed to determine the organoleptic profile and the effect of sequential co-cultures of M. pulcherrima with S. cerevisiae commercial isolates. Similar studies have demonstrated the co-inoculation potential of these two species, reducing ethanol levels and increased production of acetate esters and higher alcohols in wine [64]. This new data could provide experimental evidence to establish native M. pulcherrima as valuable resources for new local wines.

To the best of our knowledge, this is the first time where HTS has been used to describe the microbiota related to wine-environments directly from grape musts in South America. In this way, the combination of a culture-dependent method and HTS approach enabled us to estimate fungal diversity and dynamics in unfermented and fermented musts of a Sauvignon blanc variety from Chile's Central Valley. Our finding that the indigenous yeast M. pulcherrima could co-exist with a commercial S. cerevisiae strain provides support for further studies on M. pulcherrima contribution to the sensory quality of wine.

Supplementary Materials: The following are available online at http://www.mdpi.com/2076-2607/8/6/956/s1, Figure S1: PCA ordination diagram of fungal relative abundance data on M samples, Figure S2: Heatmaps for Lag phase and ODmax, Table S1: Taxonomy and average relative abundance of each OTU in M and EF samples from both seasons, Table S2: Alpha diversity. Microbial diversity indicated by Shannon diversity and Chao-1 indices, Table S3: Physicochemical parameters from unfermented musts, Table S4: Network information, Table S5: Identity, ITS region size and GenBank accession numbers of the isolated yeasts, Table S6: Kinetics parameters in all strains obtained from the microcultivation assay, Table S7: Physico-chemical characteristics of SWM for FF strains after $290 \mathrm{~h}$ of fermentation.

Author Contributions: Conceptualization, D.M., R.P., F.A.C. and V.C.; Formal analysis, D.M., J.M. and M.G.; Funding acquisition, F.A.C. and V.C.; Investigation, D.M., R.P., J.M. and W.M.; Supervision, M.G., F.A.C. and V.C.; Writing - original draft, D.M., F.A.C. and V.C.; Writing - review and editing, R.P., J.M. and M.G. All authors have read and agreed to the published version of the manuscript.

Funding: This research was funded by CORFO-Innova grant number 14IDL2-29912. 2014-2017, COPEC-PUC grant number 2014.R.297, Millennium Institute for Integrative Biology (iBio) and FONDECYT N 3190532 (to WM).

Acknowledgments: We would like to thank Viña San Pedro and especially Saddy Fuenzalida for providing the samples included in this study. The authors also thank Gabriela Gomez for technical assistance.

Conflicts of Interest: The authors declare no conflict of interest.

\section{References}

1. Fleet, G.H. Wine yeasts for the future. FEMS Yeast Res. 2008, 8, 979-995. [CrossRef] [PubMed]

2. Steensels, J.; Snoek, T.; Meersman, E.; Nicolino, M.P.; Voordeckers, K.; Verstrepen, K.J. Improving industrial yeast strains: Exploiting natural and artificial diversity. FEMS Microbiol. Rev. 2014, 38, 947-995. [CrossRef]

3. Cubillos, F.A. Exploiting budding yeast natural variation for industrial processes. Curr. Genet. 2016, 62, 745-751. [CrossRef] [PubMed]

4. Matallana, E.; Aranda, A. Biotechnological impact of stress response on wine yeast. Lett. Appl. Microbiol. 2017, 64, 103-110. [CrossRef] [PubMed]

5. Curiel, J.A.; Morales, P.; Gonzalez, R.; Tronchoni, J. Different non-Saccharomyces yeast species stimulate nutrient consumption in S. cerevisiae mixed cultures. Front. Microbiol. 2017, 8. [CrossRef]

6. Morales, P.; Rojas, V.; Quirós, M.; Gonzalez, R. The impact of oxygen on the final alcohol content of wine fermented by a mixed starter culture. Appl. Microbiol. Biotechnol. 2015, 99, 3993-4003. [CrossRef]

7. Bokulich, N.A.; Ohta, M.; Richardson, P.M.; Mills, D.A. Monitoring Seasonal Changes in Winery-Resident Microbiota. PLoS ONE 2013, 8, e0066437. [CrossRef] 
8. Bokulich, N.A.; Thorngate, J.H.; Richardson, P.M.; Mills, D.A. Microbial biogeography of wine grapes is conditioned by cultivar, vintage, and climate. Proc. Natl. Acad. Sci. USA 2014, 111. [CrossRef]

9. Pinto, C.; Pinho, D.; Cardoso, R.; Custódio, V.; Fernandes, J.; Sousa, S.; Pinheiro, M.; Egas, C.; Gomes, A.C. Wine fermentation microbiome: A landscape from different Portuguese wine appellations. Front. Microbiol. 2015, 6. [CrossRef]

10. Wei, Y.J.; Wu, Y.; Yan, Y.Z.; Zou, W.; Xue, J.; Ma, W.R.; Wang, W.; Tian, G.; Wang, L.Y. High-throughput sequencing of microbial community diversity in soil, grapes, leaves, grape juice and wine of grapevine from China. PLOS ONE 2018, 13, e0193097. [CrossRef]

11. Drumonde-Neves, J.; Franco-Duarte, R.; Lima, T.; Schuller, D.; Pais, C. Association between Grape Yeast Communities and the Vineyard Ecosystems. PLoS ONE 2017, 12, e0169883. [CrossRef] [PubMed]

12. Varela, C.; Borneman, A.R. Yeasts found in vineyards and wineries. Yeast 2017, 34, 111-128. [CrossRef] [PubMed]

13. Eder, M.L.R.; Conti, F.; Rosa, A.L. Differences between indigenous yeast populations in spontaneously fermenting musts from $V$. vinifera L. and V. labrusca L. Grapes harvested in the same geographic location. Front. Microbiol. 2018, 9. [CrossRef]

14. Bokulich, N.A.; Collins, T.S.; Masarweh, C.; Allen, G.; Heymann, H.; Ebeler, S.E.; Millsa, D.A. Associations among wine grape microbiome, metabolome, and fermentation behavior suggest microbial contribution to regional wine characteristics. MBio 2016, 7. [CrossRef] [PubMed]

15. Liu, D.; Zhang, P.; Chen, D.; Howell, K. From the Vineyard to the Winery: How Microbial Ecology Drives Regional Distinctiveness of Wine. Front. Microbiol. 2019, 10, 1-13. [CrossRef] [PubMed]

16. Padilla, B.; Gil, J.V.; Manzanares, P. Past and future of non-Saccharomyces yeasts: From spoilage microorganisms to biotechnological tools for improving wine aroma complexity. Front. Microbiol. 2016, 7, 411. [CrossRef]

17. Zhang, B.Q.; Shen, J.Y.; Duan, C.Q.; Yan, G.L. Use of indigenous Hanseniaspora vineae and Metschnikowia pulcherrima Co-fermentation with Saccharomyces cerevisiae to improve the aroma diversity of Vidal blanc icewine. Front. Microbiol. 2018, 9. [CrossRef]

18. Lombardi, S.J.; Pannella, G.; Iorizzo, M.; Moreno-Arribas, M.V.; Tremonte, P.; Succi, M.; Sorrentino, E.; Macciola, V.; Di Renzo, M.; Coppola, R. Sequential inoculum of Hanseniaspora guilliermondii and Saccharomyces cerevisiae for winemaking Campanino on an industrial scale. World J. Microbiol. Biotechnol. 2018, 34. [CrossRef]

19. Chen, K.; Escott, C.; Loira, I.; del Fresno, J.M.; Morata, A.; Tesfaye, W.; Calderon, F.; Suárez-Lepe, J.A.; Han, S.; Benito, S. Use of non-Saccharomyces yeasts and oenological tannin in red winemaking: Influence on colour, aroma and sensorial properties of young wines. Food Microbiol. 2018, 69, 51-63. [CrossRef]

20. Jara, C.; Laurie, V.F.; Mas, A.; Romero, J. Microbial terroir in chilean valleys: Diversity of non-conventional yeast. Front. Microbiol. 2016, 7. [CrossRef]

21. Ganga, M.A.; Martínez, C. Effect of wine yeast monoculture practice on the biodiversity of non-Saccharomyces yeasts. Proc. J. Appl. Microbiol. 2004, 96, 76-83. [CrossRef] [PubMed]

22. Horta, C.B.; Piccardo, C.C. Una Mirada al Mercado Vitivinícola Nacional e Internacional; Oficina de Estudios y Políticas Agrarias-Odepa: Santiago de Chile, Chile, 2018.

23. Handl, S.; Dowd, S.E.; Garcia-Mazcorro, J.F.; Steiner, J.M.; Suchodolski, J.S. Massive parallel 16S rRNA gene pyrosequencing reveals highly diverse fecal bacterial and fungal communities in healthy dogs and cats. FEMS Microbiol. Ecol. 2011, 76, 301-310. [CrossRef] [PubMed]

24. Dowd, S.E.; Callaway, T.R.; Wolcott, R.D.; Sun, Y.; McKeehan, T.; Hagevoort, R.G.; Edrington, T.S. Evaluation of the bacterial diversity in the feces of cattle using 16S rDNA bacterial tag-encoded FLX amplicon pyrosequencing (bTEFAP). BMC Microbiol. 2008, 8. [CrossRef] [PubMed]

25. Edgar, R.C.; Haas, B.J.; Clemente, J.C.; Quince, C.; Knight, R. UCHIME improves sensitivity and speed of chimera detection. Bioinformatics 2011, 27, 2194-2200. [CrossRef]

26. Edgar, R.C. Search and clustering orders of magnitude faster than BLAST. Bioinformatics 2010, 26, $2460-2461$. [CrossRef]

27. Schloss, P.D.; Westcott, S.L.; Ryabin, T.; Hall, J.R.; Hartmann, M.; Hollister, E.B.; Lesniewski, R.A.; Oakley, B.B.; Parks, D.H.; Robinson, C.J.; et al. Introducing mothur: Open-source, platform-independent, community-supported software for describing and comparing microbial communities. Appl. Environ. Microbiol. 2009, 75, 7537-7541. [CrossRef] 
28. Caporaso, J.G.; Kuczynski, J.; Stombaugh, J.; Bittinger, K.; Bushman, F.D.; Costello, E.K.; Fierer, N.; Pẽa, A.G.; Goodrich, J.K.; Gordon, J.I.; et al. QIIME allows analysis of high-throughput community sequencing data. Nat. Methods 2010, 7, 335-336. [CrossRef]

29. Nilsson, R.H.; Larsson, K.H.; Taylor, A.F.S.; Bengtsson-Palme, J.; Jeppesen, T.S.; Schigel, D.; Kennedy, P.; Picard, K.; Glöckner, F.O.; Tedersoo, L.; et al. The UNITE database for molecular identification of fungi: Handling dark taxa and parallel taxonomic classifications. Nucleic Acids Res. 2019, 47, D259-D264. [CrossRef]

30. Oksanen, J.; Blanchet, F.G.; Friendly, M.; Kindt, R.; Legendre, P.; Mcglinn, D.; Minchin, P.R.; O’hara, R.B.; Simpson, G.L.; Solymos, P.; et al. Package “Vegan"—Community Ecology Package Version 2.5-6. 2019. Available online: https://rdrr.io/cran/vegan/ (accessed on 15 January 2020).

31. Mandakovic, D.; Rojas, C.; Maldonado, J.; Latorre, M.; Travisany, D.; Delage, E.; Bihouée, A.; Jean, G.; Díaz, F.P.; Fernández-Gómez, B.; et al. Structure and co-occurrence patterns in microbial communities under acute environmental stress reveal ecological factors fostering resilience. Sci. Rep. 2018, 8, 5875. [CrossRef]

32. Faust, K.; Raes, J. CoNet app: Inference of biological association networks using Cytoscape. F1000Research 2016, 5, 1519. [CrossRef]

33. Faust, K.; Raes, J. Microbial interactions: From networks to models. Nat. Rev. Microbiol. 2012, 10, 538-550. [CrossRef] [PubMed]

34. Shannon, P.; Markiel, A.; Ozier, O.; Baliga, N.S.; Wang, J.T.; Ramage, D.; Amin, N.; Schwikowski, B.; Ideker, T. Cytoscape: A software environment for integrated models of biomolecular interaction networks. Genome Res. 2003, 13, 2498-2504. [CrossRef] [PubMed]

35. Kessi-Pérez, E.I.; Araos, S.; García, V.; Salinas, F.; Abarca, V.; Larrondo, L.F.; Martínez, C.; Cubillos, F.A. RIM15 antagonistic pleiotropy is responsible for differences in fermentation and stress response kinetics in budding yeast. FEMS Yeast Res. 2016, 16. [CrossRef] [PubMed]

36. Hall, B.G.; Acar, H.; Nandipati, A.; Barlow, M. Growth rates made easy. Mol. Biol. Evol. 2014, 31, $232-238$. [CrossRef]

37. Tapia, S.M.; Cuevas, M.; Abarca, V.; Delgado, V.; Rojas, V.; García, V.; Brice, C.; Martínez, C.; Salinas, F.; Larrondo, L.F.; et al. GPD1 and ADH3 natural variants underlie glycerol yield differences in wine fermentation. Front. Microbiol. 2018, 9. [CrossRef]

38. Rossignol, T.; Dulau, L.; Julien, A.; Blondin, B. Genome-wide monitoring of wine yeast gene expression during alcoholic fermentation. Yeast 2003, 20, 1369-1385. [CrossRef]

39. Salinas, F.; Cubillos, F.A.; Soto, D.; Garcia, V.; Bergström, A.; Warringer, J.; Ganga, M.A.; Louis, E.J.; Liti, G.; Martinez, C. The Genetic Basis of Natural Variation in Oenological Traits in Saccharomyces cerevisiae. PLOS ONE 2012, 7. [CrossRef]

40. Tesnière, C.; Delobel, P.; Pradal, M.; Blondin, B. Impact of Nutrient Imbalance on Wine Alcoholic Fermentations: Nitrogen Excess Enhances Yeast Cell Death in Lipid-Limited Must. PLoS ONE 2013, 8. [CrossRef]

41. García, V.; Rivera, J.; Contreras, A.; Ganga, M.A.; Martínez, C. Development and characterization of hybrids from native wine yeasts. Braz. J. Microbiol. 2012, 43, 482-489. [CrossRef]

42. Freilich, M.A.; Wieters, E.; Broitman, B.R.; Marquet, P.A.; Navarrete, S.A. Species co-occurrence networks: Can they reveal trophic and non-trophic interactions in ecological communities? Ecology 2018, 99, 690-699. [CrossRef]

43. De Vries, F.T.; Griffiths, R.I.; Bailey, M.; Craig, H.; Girlanda, M.; Gweon, H.S.; Hallin, S.; Kaisermann, A.; Keith, A.M.; Kretzschmar, M.; et al. Soil bacterial networks are less stable under drought than fungal networks. Nat. Commun. 2018, 9. [CrossRef] [PubMed]

44. Setati, M.E.; Jacobson, D.; Bauer, F.F. Sequence-based analysis of the Vitis vinifera L. cv cabernet sauvignon grape must mycobiome in three South African vineyards employing distinct agronomic systems. Front. Microbiol. 2015, 6. [CrossRef] [PubMed]

45. Zhang, S.; Chen, X.; Zhong, Q.; Zhuang, X.; Bai, Z. Microbial community analyses associated with nine varieties of wine grape carposphere based on high-throughput sequencing. Microorganisms 2019, 7. [CrossRef] [PubMed]

46. Duc, C.; Pradal, M.; Sanchez, I.; Noble, J.; Tesnière, C.; Blondin, B. A set of nutrient limitations trigger yeast cell death in a nitrogen-dependent manner during wine alcoholic fermentation. PLoS ONE 2017, 12. [CrossRef] [PubMed]

47. Romano, P.; Ciani, M.; Fleet, G.H. Yeasts in the Production of Wine; Springer: Basel, Switzerland, 2019. 
48. Alsammar, H.F.; Naseeb, S.; Brancia, L.B.; Gilman, R.T.; Wang, P.; Delneri, D. Targeted metagenomics approach to capture the biodiversity of Saccharomyces genus in wild environments. Environ. Microbiol. Rep. 2019, 11, 206-214. [CrossRef] [PubMed]

49. Li, J.; Hu, W.Z.; Xu, Y.P. Diversity and dynamics of yeasts during vidal blanc icewine fermentation: A strategy of the combination of culture-dependent and high-throughput sequencing approaches. Front. Microbiol. 2019, 10. [CrossRef] [PubMed]

50. Röttjers, L.; Faust, K. From hairballs to hypotheses-biological insights from microbial networks. FEMS Microbiol. Rev. 2018, 42, 761-780. [CrossRef]

51. Zhang, B.Q.; Luan, Y.; Duan, C.Q.; Yan, G.L. Use of Torulaspora delbrueckii Co-fermentation with two Saccharomyces cerevisiae Strains with different aromatic characteristic to improve the diversity of red wine aroma profile. Front. Microbiol. 2018, 9. [CrossRef]

52. Bolton, M.D.; Thomma, B.P.H.J.; Nelson, B.D. Sclerotinia sclerotiorum (Lib.) de Bary: Biology and molecular traits of a cosmopolitan pathogen. Mol. Plant Pathol. 2006, 7, 1-16. [CrossRef]

53. Williamson, B.; Tudzynski, B.; Tudzynski, P.; Van Kan, J.A.L. Botrytis cinerea: The cause of grey mould disease. Mol. Plant Pathol. 2007, 8, 561-580. [CrossRef]

54. Barata, A.; Malfeito-Ferreira, M.; Loureiro, V. The microbial ecology of wine grape berries. Int. J. Food Microbiol. 2012, 153, 243-259. [CrossRef] [PubMed]

55. Stefanini, I.; Cavalieri, D. Metagenomic approaches to investigate the contribution of the vineyard environment to the quality of wine fermentation: Potentials and difficulties. Front. Microbiol. 2018, 9, 991. [CrossRef] [PubMed]

56. Auld, R.R.; Myre, M.; Mykytczuk, N.C.S.; Leduc, L.G.; Merritt, T.J.S. Characterization of the microbial acid mine drainage microbial community using culturing and direct sequencing techniques. J. Microbiol. Methods 2013, 93, 108-115. [CrossRef] [PubMed]

57. Romão, D.; Staley, C.; Ferreira, F.; Rodrigues, R.; Sabino, R.; Veríssimo, C.; Wang, P.; Sadowsky, M.; Brandão, J. Next-generation sequencing and culture-based techniques offer complementary insights into fungi and prokaryotes in beach sands. Mar. Pollut. Bull. 2017, 119, 351-358. [CrossRef]

58. Stefani, F.O.P.; Bell, T.H.; Marchand, C.; De La Providencia, I.E.; El Yassimi, A.; St-Arnaud, M.; Hijri, M. Culture-dependent and -independent methods capture different microbial community fractions in hydrocarbon-contaminated soils. PLoS ONE 2015, 10. [CrossRef]

59. Crépin, L.; Sanchez, I.; Nidelet, T.; Dequin, S.; Camarasa, C. Efficient ammonium uptake and mobilization of vacuolar arginine by Saccharomyces cerevisiae wine strains during wine fermentation. Microb. Cell Fact. 2014, 13, 109. [CrossRef]

60. Gutiérrez, A.; Sancho, M.; Beltran, G.; Guillamon, J.M.; Warringer, J. Replenishment and mobilization of intracellular nitrogen pools decouples wine yeast nitrogen uptake from growth. Appl. Microbiol. Biotechnol. 2016, 100, 3255-3265. [CrossRef]

61. Jolly, N.P.; Varela, C.; Pretorius, I.S. Not your ordinary yeast: non- Saccharomyces yeasts in wine production uncovered. FEMS Yeast Res. 2014, 14, 215-237. [CrossRef]

62. Lencioni, L.; Romani, C.; Gobbi, M.; Comitini, F.; Ciani, M.; Domizio, P. Controlled mixed fermentation at winery scale using Zygotorulaspora florentina and Saccharomyces cerevisiae. Int. J. Food Microbiol. 2016, 234, 36-44. [CrossRef]

63. Lencioni, L.; Taccari, M.; Ciani, M.; Domizio, P. Zygotorulaspora florentina and Starmerella bacillaris in multistarter fermentation with Saccharomyces cerevisiae to reduce volatile acidity of high sugar musts. Aust. J. Grape Wine Res. 2018, 24, 368-372. [CrossRef]

64. Hranilovic, A.; Gambetta, J.M.; Jeffery, D.W.; Grbin, P.R.; Jiranek, V. Lower-alcohol wines produced by Metschnikowia pulcherrima and Saccharomyces cerevisiae co-fermentations: The effect of sequential inoculation timing. Int. J. Food Microbiol. 2020, 329, 108651. [CrossRef] [PubMed]

(C) 2020 by the authors. Licensee MDPI, Basel, Switzerland. This article is an open access article distributed under the terms and conditions of the Creative Commons Attribution (CC BY) license (http://creativecommons.org/licenses/by/4.0/). 